\title{
Willibald SteinMetz
}

\section{Vierzig Jahre Begriffsgeschichte - The State of the Art}

\begin{abstract}
Der Aufsatz bietet zunächst einen Überblick über neuere Tendenzen und offene Fragen der internationalen begriffsgeschichtlichen Forschung und plädiert für Historische Semantik als Disziplinbezeichnung für das sich über die klassische Begriffsgeschichte hinaus erweiternde Feld. Gefragt wird sodann nach Erklärungsmodellen für semantischen Wandel in der Geschichte. Drei Modelle von Wandel werden genauer erörtert: Plausibilitätsverlust von Redeweisen durch überraschende Ereignisse und Umbrüche, Zunahme des strategischen Gebrauchswerts von Redeweisen in wiederkehrenden Kommunikationssituationen, Irritation des Wort- und Bedeutungshaushalts einer Sprache durch Wortimporte aus einer anderen Sprache. Ausgehend vom letztgenannten Modell werden abschließend Theorieprobleme diskutiert, die sich aus der Forderung nach einer transnationalen bzw. vergleichenden historischen Semantik ergeben.
\end{abstract}

\section{Neue Forschungsfelder: von der Begriffsgeschichte zur Historischen Semantik}

Eine Bestandsaufnahme gegenwärtiger Praxis und offener Fragen der Begriffsgeschichte scheint nur sinnvoll, wenn sie als Forschungsrichtung eine Zukunft besitzt. Diese ist ihr jüngst von einem ihrer prominenten Vertreter der älteren Generation, Hans Ulrich Gumbrecht, abgesprochen worden. An seinem Schreibtisch sitzend, den Blick auf die in den Regalen rings um ihn aufgebauten Monumentalwerke begriffshistorischer Arbeit gerichtet - das „Historische Wörterbuch der Philosophie“, die "Geschichtlichen Grundbegriffe“, das „Handbuch politisch-sozialer Grundbegriffe in Frankreich“, die „Ästhetischen Grundbegriffe“ -, kündet Gumbrecht von einer „Versteinerung der lebhaften begriffsgeschichtlichen Arbeit".. Die lexikalischen Großprojekte, an denen er selbst mitgewirkt hat, erscheinen ihm als „Pyramiden des Geistes“, als „Zeugnisse aus einer abgeschlossenen Epoche der Geisteswissenschaften". ${ }^{2}$ Die Begriffsgeschichte also - ein Totenreich?

1 Hans Ulrich Gumbrecht: Pyramiden des Geistes. Über den schnellen Aufstieg, die unsichtbaren Dimensionen und das plötzliche Abebben der begriffsgeschichtlichen Bewegung. In: ders: Dimensionen und Grenzen der Begriffsgeschichte. München 2006. S. $7-36$, S. 9.

2 Ebd., S. 7. 
Vielleicht haben die lexikalischen Monumentalwerke Gumbrecht den Blick etwas verstellt. Jedenfalls scheint ihm verborgen geblieben zu sein, dass sich die begriffsgeschichtliche Arbeit in kräftiger Bewegung befindet, gerade auch außerhalb Deutschlands. Als ,ein spezifisch deutsches Anliegen und Programm" ${ }^{3}$ kann Begriffsgeschichte schon seit mindestens zehn Jahren nicht mehr gelten. Es genügt, an einer der seit 1998 jährlich stattfindenden Konferenzen der "History of Political and Social Concepts Group" teilzunehmen, um dies zu bemerken. ${ }^{4}$ Vorrangig diskutiert wird hier die historiographische Begriffsgeschichte, wie sie von Reinhart Koselleck konzipiert und von Rolf Reichardt und anderen weitergeführt wurde, während die philosophische Begriffsgeschichte im Umkreis des „Historischen Wörterbuchs der Philosophie“, wohl wegen ihrer schwächeren Verankerung in der allgemeinen Sozial-, Politik- und Mentalitätsgeschichte, bisher außerhalb des deutschen Sprachraums weniger inspirierend gewirkt hat. ${ }^{5}$ Wie man auf den Konferenzen des internationalen und interdisziplinären Forschernetzwerks der „History of Concepts Group" beobachten kann, ist Begriffsgeschichte in der Nachfolge Kosellecks eine der wenigen Hervorbringungen deutscher Geisteswissenschaften, die derzeit weltweit beachtet und kritisch fortentwickelt werden. Die internationale Aneignung schlägt sich in vielgestaltigen Publikationen nieder. So gibt es inzwischen neben dem ehrwürdigen „Archiv für Begriffsgeschichte" (seit 1955) und den eher wort- als begriffsgeschichtlich ausgerichteten "Cahiers de Lexicologie“ (seit 1959) zwei neue englischsprachige Zeitschriften mit internationalen Herausgebergremien, die dezidiert der begriffsgeschichtlichen Theorie- und Methodendiskussion als Foren dienen und empirische Beiträge publizieren: das in Finnland redigierte Jahrbuch „Redescriptions. Yearbook of Political Thought and Conceptual History“ (seit 1997) ${ }^{6}$ und die von Brasilien aus koordinierte Zeitschrift „Contributions to the History of Concepts“ (seit 2005). ${ }^{7}$ Für zahlreiche Länder beziehungsweise Sprachräume der Erde sind untereinander vernetzte Forschergruppen mit

3 Ebd., S. 9.

4 Über die Konferenzen und Aktivitäten der Gruppe informieren die Internetseiten http://www.jyu.fi/yhtfil/hpscg/ (Zugriff am 1. Mai 2007).

5 Vgl. die Beurteilung von Melvin Richter: The History of Political and Social Concepts. A Critical Introduction, New York/Oxford 1995. Die Kritik bezieht sich vor allem auf die - im Vergleich zu den „Geschichtlichen Grundbegriffen“ noch ungleich größere Verknappung aller Hinweise auf Kontexte und pragmatische Funktionen des Wortgebrauchs im „Historischen Wörterbuch der Philosophie“. Die durch das Format des Wörterbuchs erzwungenen Begrenzungen werden in der interdisziplinären Fachdiskussion zunehmend als Belastung empfunden; vgl. die Beiträge in: Gunter Scholtz $(\mathrm{Hg}$.): Die Interdisziplinarität der Begriffsgeschichte. (Archiv für Begriffsgeschichte, Sonderheft). Hamburg 2000; Ernst Müller (Hg.): Begriffsgeschichte im Umbruch? (Archiv für Begriffsgeschichte, Sonderheft). Hamburg 2005.

6 Das Jahrbuch erschien von 1997 bis 2002 unter dem Titel „Finnish Yearbook of Political Thought", seit 2003 erscheint es unter dem jetzigen Titel.

7 Die Zeitschrift ist sowohl in gedruckter Forma als auch im Internet verfügbar, vgl. http://contributions.iuperj.br/ (Zugriff am 1. Mai 2007). 
nationalen, vergleichenden und transfergeschichtlichen begriffsgeschichtlichen Forschungen beschäftigt. Dies geschieht teils in Form von Monographien und Aufsatzsammlungen, teils in Form gröBerer lexikalischer Projekte, die sich am Vorbild der „Geschichtlichen Grundbegriffe“ und des „Handbuchs politischsozialer Grundbegriffe in Frankreich" orientieren, dabei jedoch sinnvollerweise kleiner dimensioniert sind. Zahlreiche monographische und kleinere Einzelstudien zu Begriffen und Begriffsnetzen liegen beispielsweise für die skandinavischen Länder vor, zum Teil in vergleichender Perspektive. ${ }^{8}$ Größere Sammelwerke sind für Frankreich, Spanien und die südamerikanischen Länder in Arbeit. ${ }^{9}$ In den Niederlanden befasst sich seit vielen Jahren eine Forschergruppe mit der Geschichte niederländischer politisch-sozialer Grundbegriffe und ihrer Beziehung zu den drei wichtigsten Nachbarsprachen (Französisch, Deutsch, Englisch) sowie zur gemeinsamen lateinischen Tradition. ${ }^{10}$ In jüngster Zeit schlagen sich auch die Diskussionen zwischen deutschen und russischen Begriffshistorikern in ersten Publikationen nieder; Ost-

8 Exemplarisch seien genannt: Norbert Götz: Ungleiche Geschwister. Die Konstruktion von nationalsozialistischer Volksgemeinschaft und schwedischem Volksheim. BadenBaden 2001; Jussi Kurunmäki: Representation, Nation and Time. The Political Rhetoric of the 1866 Parliamentary Reform in Sweden. Jyväskylä 2000; Jonas Harvard: En helig allmännelig opinion. Föreställingar om offentlighet och legitimitet i svensk riksdagsdebatt 1848-1919. Umeå 2006; Kari Palonen: Transforming a Common European Concept to Finnish: Conceptual Changes in the Understanding of Politiikka. In: Finnish Yearbook of Political Thought 5 (2001), S. 113-153.

9 Für Frankreich: Rolf Reichardt/Hans-Jürgen Lüsebrink $(\mathrm{Hg}$.$) : Handbuch politisch-$ sozialer Grundbegriffe in Frankreich 1680-1920, bisher 20 Hefte, München $1985 \mathrm{ff}$;; Dictionnaire des usages socio-politiques, bisher 8 Hefte (verschiedene Herausgeber), Paris 1985 ff;; als Beispiel für eine historisch-semantische Arbeit zum 20. Jahrhundert: Damon Mayaffre: Le poids des mots. Le discours de gauche et de droite dans l'entre deux-guerres. Maurice Thorez, Léon Blum, Pierre-Étienne Flandin et André Tardieu (1928-1939). Paris 2000; für Spanien: Javier Fernández Sebastián/Juan Francisco Fuentes (Hg.): Diccionario Político y Social del Siglo XIX Español. Madrid 2002; zum brasilianischen Projekt: João Feres Júnior: For a Critical Conceptual History of Brazil: Receiving Begriffsgeschichte. In: Contributions to the History of Concepts 1 (2005), S. 185-200; für erste Informationen zum geplanten Projekt „Iberconceptos. Proyecto Iberoamericano de Historia Conceptual" siehe http://foroiberoideas. cervantesvirtual.com/ (Zugriff am 14. Mai 2007).

10 Vgl. Karin Tilmans: Applying Begriffsgeschichte to Dutch History. Some Remarks on the Practice and Future of a Project. In: Contributions to the History of Concepts 2 (2006), S. 43 58; Pim den Boer: The Historiography of German Begriffsgeschichte and the Dutch Project of Conceptual History. In: Iain Hampsher-Monk/Karin Tilmans/ Frank van Vree ( $\mathrm{Hg}$.): History of Concepts: Comparative Perspectives. Amsterdam 1998. S. 13-22. Eine Reihe von Arbeiten zu niederländischen Begriffen sind bereits erschienen: N.C. F. van Sas (Hg.): Vaderland. Een geschiedenis van de vijftiende eeuw tot 1940. Amsterdam 1999; E. O. G. Haitsma Mulier/W. R. E. Velema (Hg.): Vrijheid. Een geschiedenis van de vijftiende tot de twintigste eeuw. Amsterdam 1999; Pim den Boer ( $\mathrm{Hg}$.): Beschaving. Een geschiedenis va de begrippen hoofsheid, heusheid, beschaving en cultuur. Amsterdam 2001; Joost Kloek/Karin Timans (Hg.): Burger. Een geschiedenis van het begrip , burger' in de Nederlanden van de Middeleeuwen tot de $21^{\text {ste }}$ eeuw. Amsterdam 2002. 
europa insgesamt dürfte nicht mehr lange begriffsgeschichtlich eine terra incognita bleiben. ${ }^{11}$ Auf der letzten Konferenz der erwähnten „History of Political and Social Concepts Group" in Uppsala (2006) ist über die Aneignung und Umsemantisierung westlicher Begriffe in China und Korea im 19. und 20. Jahrhundert diskutiert worden. ${ }^{12}$ Ähnliche Probleme der Übersetzung und Übersetzbarkeit westlicher sozialer Beschreibungs- und Klassifikationsbegriffe (,Klasse', ,Kaste', ,Bürger') in die Sprachen der indo-islamischen Welt (und umgekehrt) im kolonialen und postkolonialen Zeitalter sind ebenfalls in den letzten Jahren Gegenstand der Forschung geworden. ${ }^{13}$

Die Begriffsgeschichte ist also, das zeigt der geraffte Überblick, international mit starker deutscher Beteiligung sehr lebendig. Die Lebendigkeit äußert sich auch darin, dass die genannten Forschergruppen sich nicht damit begnügen, das in Deutschland etablierte Paradigma der lexikalisch-semasiologisch verfahrenden Begriffsgeschichte auf bisher unerforschte Länder und Sprachen lediglich zu übertragen. Vielmehr überprüfen sie es kritisch, passen es an, stellen neue Fragen. Gerade die von Gumbrecht sehr zu Recht als ungelöst angesprochenen Probleme der Begriffsgeschichte gewinnen an Brisanz, je mehr sich die Begriffsgeschichte internationalisiert: Das häufige Fehlen direkt äquivalenter Begriffe in anderen Sprachen lässt die Frage dringlich werden, auf welchen Wegen nach metaphorischen, aber auch umgangssprachlichen und visuellen Artikulationen der noch nicht oder nicht mehr begrifflich ge-

1 Vgl. Peter Thiergen (Hg.): Russische Begriffsgeschichte der Neuzeit. Beiträge zu einem Forschungsdesiderat. Köln 2006; Nikolaj E. Koposov (Hg.): Istoričeskie ponjatija i političeskie idei v Rossii XVI-XX veka. [Geschichtliche Begriffe und politische Ideen in Russland, 16.-20. Jahrhundert.]. Sankt-Petersburg 2006; dazu auch die Beiträge von Michail Krom, Ingrid Schierle und Walter Sperling zum russischen Politikbegriff vom 17. bis zum 20. Jahrhundert in: Willibald Steinmetz (Hg.): ,Politik“. Situationen eines Wortgebrauchs im Europa der Neuzeit. Frankfurt/New York 2007, S. 206-288. Zahlreiche Studien zur Geschichte einzelner russischer Begriffe und zur politischen Sprache Russlands allgemein auch in der seit 2000 erscheinenden, gedruckt und im Internet publizierten Zeitschrift „Ab Imperio“, vgl:: http://abimperio.net/ (Zugriff am 1. Mai 2007).

12 Zur Erforschung der chinesischen Begriffswelt im Vergleich das von Christoph Harbsmeier (Oslo) geleitete Projekt: Thesaurus Linguae Sericae (TLS). An Historical and Comparative Encyclopaedia of Chinese Conceptual Schemes: http:/www.hf.uio.no/ forskningsprosjekter/tls/index.html. Dazu Christoph Harbsmeier: Concepts that make Multiple Modernities: The Conceptual Modernisation of China in a Historical and Critical Perspective, zugänglich über http://www.hf.uio.no/forskningsprosjekter/tls/ publications/index.html (Zugriff am 14. Mai 2007).

13 Hierzu grundlegend: Margrit Pernau: Bürger mit Turban. Muslime in Delhi im 19. Jahrhunderts, Habilitationsschrift Bielefeld 2006. (angekündigt Göttingen 2007); dies.: Gab es eine indische Zivilgesellschaft im 19. Jahrhundert? Überlegungen zum Verhältnis von Globalgeschichte und historischer Semantik. Erscheint in: Traverse Heft 3 (2007); Monica Juneja/Margrit Pernau: Lost in Translation. Transkulturelle Geschichte und das Problem der universellen Begriffe. In: Jürgen Kocka/Heinz-Gerhard Haupt (Hg.): Beyond Comparison. (angekündigt New York/Oxford 2007). 
fassten Weltwahrnehmung gesucht werden kann. ${ }^{14}$ Ebenso stellt sich das Problem der Konzeptualisierung des Bedingungsverhältnisses zwischen Sprachgebrauch, Begriffsbildung und sogenannter ,Sachgeschichte' noch einmal komplexer dar, wenn zwischen verschiedenen Sprachgemeinschaften Vergleiche angestellt und Übersetzungsvorgänge zwischen ihnen untersucht werden. ${ }^{15}$ Die Herausforderungen, die sich der Begriffsgeschichte im Zuge ihrer Internationalisierung stellen, gehören zu den spannendsten Forschungsfeldern der Geschichtswissenschaft überhaupt. Dass sie nur in Zusammenarbeit mit der Linguistik und anderen Nachbardisziplinen bearbeitet werden können, versteht sich von selbst.

Nicht nur international entwickelt sich die Begriffsgeschichte weiter, auch innerhalb des deutschen Sprachraums wendet sie sich neuen thematischen Feldern zu, erschließt sich neue Zeiträume und nutzt neue Methoden. Als Beispiel für ein thematisches Feld, das derzeit in der Geschichtswissenschaft besondere Aufmerksamkeit findet, sei nur das Feld der religiösen Begrifflichkeiten genannt. Eine Bochumer Forschergruppe um Lucian Hölscher befasst sich mit den sprachlichen Ausdrucksformen religiöser Vergemeinschaftung in der europäischen Geschichte seit der Frühen Neuzeit. ${ }^{16}$ Die Forschungen reichen von der Geschichte des Religionsbegriffs selbst über die Geschichte der wechselseitigen Selbst- und Fremdidentifizierungen von Religionsgrup-

I4 Gumbrecht, Pyramiden [wie Anm. 1], S. 15 f. u. 35 f. erörtert das Problem, wie man sich dem Vor- und Unbegrifflichen annähern kann, lediglich mit Blick auf die Metaphorologie im Anschluss an Hans Blumenberg. Daneben ist aber auch die Suche nach umgangssprachlichen Umschreibungen sowie Visualisierungen als Zugang zum Vorbeziehungsweise Unbegrifflichen zu nennen. Zu Suchstrategien nach dem Vorbegrifflichen am Beispiel des Begriffs ,Fremdherrschaft': Christian Koller: Fremdherrschaft. Ein politischer Kampfbegriff im Zeitalter des Nationalismus. Frankfurt/Main 2005. S. 32 f. u. 131-190. Die Metaphorologie ist im übrigen von den Vertretern der philosophischen Begriffsgeschichte nicht so vernachlässigt worden, wie Gumbrecht behauptet. Vor allem die theoretischen und empirischen Arbeiten Ralf Konersmanns sind hier zu erwähnen: Ralf Konersmann: Metaphorisches Wissen. In: ders./Peter Noever/Peter Zumthor: Zwischen Bild und Realität. Zürich 2006. S. 10-37; ders.: Figuratives Wissen. Zur Konzeption des Wörterbuchs der philosophischen Metaphern. In: Neue Rundschau 116 (2005), H. 2, S. 19-35; ders. (Hg.): Wörterbuch der philosophischen Metaphern. Darmstadt 2007. Vgl. außerdem: Ruben Zimmermann: Einführung: Bildersprache verstehen oder: Die offene Sinndynamik der Sprachbilder. In: ders. (Hg.): Bildersprache verstehen. Zur Hermeneutik der Metapher und anderer bildlicher Sprachformen. Mit einem Geleitwort von Hans-Georg Gadamer. München 2000. S. 13-54. Ein intensiv bearbeitetes Forschungsfeld ist die Metaphernzirkulation zwischen Naturwissenschaften (Medizin) und Politik; vgl.: Philipp Sarasin: Infizierte Körper, kontaminierte Sprachen. Metaphern als Gegenstand der Wissenschaftsgeschichte. In: ders.: Geschichtswissenschaft und Diskursanalyse. Frankfurt/Main 2003. S. 191230.

$15 \mathrm{Zu}$ Recht stellt Gumbrecht, Pyramiden, S. 27, fest, dass eine „Unentschiedenheit im Hinblick auf das Problem der Weltreferenz von Sprache" (Hervorhebung im Original) die Begriffsgeschichte Kosellecks immer gekennzeichnet habe.

16 Vgl. jüngst: Lucian Hölscher (Hg.): Baupläne der sichtbaren Kirche. Sprachliche Konzepte religiöser Vergemeinschaftung in Europa. Göttingen 2007. 
pen bis hin zu den Bezeichnungen für religiöse Institutionen und Praktiken (,Kirche', ,Frömmigkeit', ,Glaube'). Angesichts der Zunahme religiös begründeter Konflikte in der Gegenwart gewinnen diese begriffsgeschichtlichen Forschungen zur religiösen Semantik zunehmend auch eine politisch aufklärende Dimension.

Was die neu zu erschließenden Zeiträume betrifft, ist vor allem das 20. Jahrhundert aufgrund der bekannten Konzentration des Lexikons „Geschichtliche Grundbegriffe" auf die sogenannte "Sattelzeit" bisher von historischer Seite nur stiefmütterlich behandelt worden. ${ }^{17}$ Von linguistischer Seite sind hingegen umfangreiche Arbeiten zum öffentlichen Sprachgebrauch nach 1945, zum Teil in lexikographischer Form, vorgelegt worden. ${ }^{18}$ Daran haben alle weiteren Forschungen anzuknüpfen, gleich ob sie im engeren Sinne begriffsgeschichtlich verfahren ${ }^{19}$, oder ob sie ein weiteres Verständnis von historischer Diskursanalyse zugrunde legen, das sich an Michel Foucault oder anderen Theoretikern orientiert. ${ }^{20}$ Für gegenwartsbezogene Untersuchungen, beispielsweise zu den „Semantiken des Wohlfahrtsstaats“, sind außer Koselleck und Foucault auch Niklas Luhmanns Studien zum Verhältnis von Gesellschaftsstruktur und Semantik richtungsweisend geworden. ${ }^{21}$

17 Brauchbare Arbeiten zur Geschichte einzelner Begriffe liegen aber für kürzere Zeitabschnitte durchaus vor; vgl. beispielsweise für den Politikbegriff: Sabine Marquardt: Polis contra Polemos. Politik als Kampfbegriff der Weimarer Republik. Köln 1997; Kari Palonen: Politik als Handlungsbegriff. Horizontwandel des Politikbegriffs in Deutschland 1890-1933; ders.: The Struggle with Time. A Conceptual History of ,Politics' as an Activity. Hamburg 2006; sowie mehrere Beiträge in Steinmetz (Hg.), ,Politik‘ [wie Anm. 11].

18 Exemplarisch seien genannt: Georg Stötzel/Martin Wengeler $(\mathrm{Hg}$.): Kontroverse Begriffe. Geschichte des öffentlichen Sprachgebrauchs in der Bundesrepublik Deutschland. Berlin/New York 1994; Karin Böke/Frank Liedtke/Martin Wengeler: Politische Leitvokabeln in der Adenauer-Ära. Berlin/New York 1996; Matthias Jung/Thomas Niehr/Karin Böke: Ausländer und Migranten im Spiegel der Presse. Ein diskurshistorisches Wörterbuch zur Einwanderung seit 1945. Wiesbaden 2000; Georg Stötzel/ Thorsten Eitz (Hg.): Zeitgeschichtliches Wörterbuch der deutschen Gegenwartssprache. Hildesheim 2002; Heidrun Kämper: Der Schulddiskurs in der frühen Nachkriegszeit. Ein Beitrag zur Geschichte des sprachlichen Umbruchs nach 1945. Berlin/New York 2005; dies.: Opfer - Täter - Nichttäter. Ein Wörterbuch zum Schulddiskurs 1945-1955. Berlin/New York 2007.

19 Ausdrücklich an die „Geschichtlichen Grundbegriffe" anschließend, jedoch mit theoretischen Beiträgen zu ihrer Erweiterung und empirischen Studien zu wichtigen Begriffen der Nachkriegszeit: Carsten Dutt (Hg.): Herausforderungen der Begriffsgeschichte. Heidelberg 2003.

20 Hierzu im Überblick: Jacques Guilhaumou: Geschichte und Sprachwissenschaft - Wege und Stationen (in) der, analyse du discours‘. In: Reiner Keller/Andreas Hirseland/ Werner Schneider/Willy Viehöfer (Hg.): Handbuch Sozialwissenschaftliche Diskursanalyse. Bd. II: Forschungspraxis. Opladen 2003. S. 19-65; vgl. dort auch die Anwendungsbeispiele, überwiegend zum 20. Jahrhundert.

21 Vgl. Stephan Lessenich: Einleitung: Wohlfahrtsstaatliche Grundbegriffe - Semantiken des Wohlfahrtsstaats. In: ders. (Hg.): Wohlfahrtsstaatliche Grundbegriffe: Historische und aktuelle Diskurse. Frankfurt/New York 2003. S. 9-19; dazu auch: ders.: Schluss: Wohlfahrtsstaatliche Semantiken - Politik im Wohlfahrtsstaat. In: ebd.: S. 419-426; 
Neben dem 20. Jahrhundert ist die europäische Vormoderne, also die lange Epoche vom Ende des Weströmischen Reichs bis zur Mitte des 18. Jahrhunderts ein Zeitraum, der aus begriffshistorischer Sicht noch weithin unerschlossenes Gelände bietet. Schwierig und zugleich faszinierend ist diese Epoche für Begriffshistoriker und -historikerinnen vor allem deshalb, weil wir es hier überall in Europa mit Gesellschaften zu tun haben, in denen Mehrsprachigkeit auf einem Territorium - sozial und funktional differenziert - der Normalfall war. Es ist für diesen Zeitraum noch weniger als für die Moderne möglich, ,nationale' Sprachgemeinschaften künstlich voneinander zu isolieren. ${ }^{22}$ Mindestens die dauernden Übersetzungen und Rückübersetzungen zwischen lateinischer (schriftlicher) Gelehrtensprache und volkssprachlicher (mündlicher, zunehmend verschriftlichter) Praxis in Politik, Kirche, Rechtsprechung und anderen Handlungsfeldern müssen systematisch, nicht nur punktuell berücksichtigt werden. Vom 17. bis ins frühe 19. Jahrhundert kam dazu noch eine ähnliche sprachliche Wechselbeziehung zwischen einem häufig noch wenig elaborierten Gebrauch der Vernakularsprache und einer Elitenkommunikation, die fremdsprachlich geführt wurde: vornehmlich auf Französisch, in nord- und osteuropäischen Ländern aber auch auf Deutsch oder in anderen Sprachen, in Finnland beispielsweise Schwedisch. ${ }^{23}$ Es fehlt für diese langen Phasen europäischer Mehrsprachigkeit nicht nur an begriffshistorischen Einzelstudien zu Übersetzungsprozessen zwischen Gelehrten-, Eliten- und Volkssprache, sondern auch an einem analytischen Bezugsrahmen, der - ähnlich wie Kosellecks zu heuristischen Zwecken eingeführte Sattelzeithypothese - geeignet wäre, dahingehenden Forschungen eine Richtung zu geben. ${ }^{24}$

die Beiträge des Bandes schwanken zwischen eher theoretischen Erörterungen und Analysen zum Gebrauch von Leitvokabeln.

22 Im Uberblick hierzu: Peter Burke: Languages and Communities in Early Modern Europe. Cambridge 2004.

$23 \mathrm{Vgl}$. jetzt als Fallstudie zur Funktion, zum Prestige und Gebrauch von Fremdsprachen, vor allem Französisch, am Beispiel Brandenburg-Preussens: Volker Wittenauer: Im Dienste der Macht. Kultur und Sprache am Hof der Hohenzollern. Vom Großen Kurfürst bis zu Wilhelm II. Paderborn 2007.

24 Vorerst gibt es nur Einzelstudien zur Wechselbeziehung zwischen Volks- und Gelehrtensprache in bestimmten Textgattungen und Handlungskontexten; zur lateinischen, deutschen und italienischen Bürgerbegrifflichkeit z. B.: Ulrich Meier: Burgerlich vereynung. Herrschende, beherrschte und, mittlere' Bürger in Politiktheorie, chronikalischer Überlieferung und städtischen Quellen des Spätmittelalters. In: Klaus Schreiner/Reinhart Koselleck ( $\mathrm{Hg}$.): Bürgerschaft. Rezeption und Innovation der Begrifflichkeit vom Hohen Mittelalter bis ins 19. Jahrhundert. Stuttgart 1994. S. 274-306; ders.: Mensch und Bürger. Die Stadt im Denken spätmittelalterlicher Theologen, Philosophen und Juristen. München 1994. Zum lateinischen und französischen Politikvokabular: Olivier Bertrand: Le vocabulaire politique aux XIV $\mathrm{X}^{\mathrm{e}}$ et $\mathrm{XV}^{\mathrm{e}}$ siècles: constitution d'un lexique ou émergence d'une science? In: Langage \& Société, Nr. 113, Sept. 2005, S. 11-32. Zum lateinischen und schwedischen politisch-sozialen Vokabular in der Frühen Neuzeit: Bo Lindberg: Den antika skevheten. Politiska ord och begrepp i det tidig-moderna Sverige. Stockholm 2006; den Hinweis auf Lindbergs Arbeit verdanke ich einem unveröffentlichten Tagungspapier von Jani Marjanen (Helsinki): The History of Concepts beyond 
Die den Eliten angehörenden Leser(innen), Sprecher(innen) und Schreiber(innen) mehrerer Sprachen standen in diesen Jahrhunderten des Übergangs zu national homogeneren Sprachgemeinschaften vor der Herausforderung, im Alltag ständig die Sprache wechseln zu müssen, dauernd Bedeutungen abgleichen zu müssen. Umgekehrt ergab sich für sie daraus auch die Chance, mit den noch wenig fixierten Wortbedeutungen in größerem Maße zu ,spielen' als es später der Fall war, nachdem in Frankreich, Deutschland und anderen Ländern die Bemühungen um Sprachnormierung erste Erfolge erzielt hatten. Zwischen der Explosion volkssprachlicher Schriftzeugnisse im Spätmittelalter und den allmählich Wirkung zeitigenden Versuchen, Wortbedeutungen durch ein-, zwei- oder mehrsprachige Lexika wenigstens für gewisse Zeit zu fixieren, ist mithin, so könnte eine vorläufige Hypothese lauten, von einer dauernden Instabilität oder gar Unklarheit von Wortbedeutungen in den Vernakularsprachen als Regelfall auszugehen. Begriffsgeschichte wird dadurch erheblich komplizierter. Geeignete methodische Instrumente, um mit diesem Problem umzugehen, sind im Rahmen der Historischen Semantik noch zu entwickeln; ein bloß semasiologisch verfahrendes, an Einzelwörtern orientiertes Vorgehen, so viel steht fest, scheint hier weniger erfolgversprechend als es für die Epoche der ,Sattelzeit" - trotz aller berechtigter Kritik am Konzept der "Geschichtlichen Grundbegriffe“ - letztlich doch gewesen ist.

Was nun das im engeren Sinne ,lateinische“ Mittelalter angeht, also die Jahrhunderte bis etwa 1200 , in denen sich die schriftliche Kommunikation ausschließlich oder ganz überwiegend nur des Lateinischen bediente, scheint es hier wiederum etwas leichter zu sein, dem klassischen begriffsgeschichtlichen Paradigma zu folgen. ${ }^{25}$ Forschungen zum politisch-sozialen und - damit untrennbar verbunden - religiösen Sprachgebrauch zwischen Spätantike und Hochmittelalter können außerdem davon profitieren, dass schon jetzt ein beträchtlicher Teil der kanonisierten theologischen und damit auch politischtheoretischen Texte in digitalisierter Form vorliegt. Für einfache Fragestellungen wie etwa die Bestimmung sich wandelnder Verwendungszusammenhänge von Wörtern reduziert sich damit der Aufwand der Belegstellensuche um ein Vielfaches; dies vor allem dann, wenn es gelingen wird, die schon be-

the Nation-State? Discourses on Methodological Nationalism and Histoire Croisée as New Challenges. Bielefeld 2007.

25 Das heißt selbstverständlich nicht, dass nicht auch unter Mediävisten die Forderung erhoben wird, von der nur wortbezogenen Historischen Semantik zu einer satz- oder textbezogenen Historischen Semantik überzugehen; vgl. Ludolf Kuchenbuch: ,Feudalismus': Versuch über die Gebrauchsstrategien eines wissenspolitischen Reizwortes. In: Natalie Fryde/Pierre Monnet/Otto Gerhard Oexle (Hg.): Die Gegenwart des Feudalismus/Présence du féodalisme et présent de la féodalité/The Presence of Feudalism. Göttingen 2002. S. 293-323; hier S. 314 f;; ein Beispiel für eine Untersuchung, die zumindest ein ganzes Wortfeld (und die zugehörigen Praktiken) abdeckt: ders.: Porcus donativus. Language Use and Gifting in Seignorial Records between the Eigth and the Twelfth Centuries. In: Gadi Algazi/Valentin Groebner/Bernhard Jussen (Hg.): Negotiating the Gift. Pre-Modern Figurations of Exchange. Göttingen 2003. S. 193-246. 
stehenden computergestützten Verfahren der Lemmatisierung und Kollokationsanalyse weiter zu optimieren. ${ }^{26}$ Die bisher stark auf synchrone Analysen der Gegenwartssprache fixierte Korpuslinguistik und Texttechnologie muss dafür allerdings erst für diachrone Untersuchungen sensibilisiert und zur Entwicklung entsprechender, für die historische Forschung brauchbarer Erkennungs-, Unterscheidungs- und Abfrageverfahren animiert werden. Umgekehrt erfordert dies von den Historikerinnen und Historikern, ihre Terminologie und Methodik linguistischen Genauigkeitsstandards anzupassen, andernfalls wird die Historische Semantik von den Vorteilen der neuen Technologien kaum profitieren können..$^{27}$

Die wenigen Bemerkungen zu neuen Forschungsfeldern mögen genügen, um Gumbrechts Eindruck, die Begriffsgeschichte sei tot, zu widerlegen. Zutreffend wäre eine solche Annahme allenfalls, wenn man das Forschungsprogramm, das vor vierzig Jahren in den Schriften Reinhart Kosellecks und anderer umrissen und seitdem fortentwickelt wurde, mit der Vollendung des Lexikons „Geschichtliche Grundbegriffe" bereits als erfüllt ansähe. Koselleck selbst wäre der letzte gewesen, der das zugegeben hätte. Er war, je länger die Arbeit am Lexikon sich hinzog, desto unzufriedener mit der praktischen Umsetzung seiner theoretischen Konzeptionen, und er war sich der Defizite, die der spezifischen lexikalischen Darstellungsform geschuldet waren, vollkommen bewusst. Er sprach von der "theoretischen Zwangsjacke“, die der einmal gewählte Zuschnitt des Lexikons für ihn bedeutete. ${ }^{28}$ Das Lexikon war für ihn nur ein erster Schritt auf dem Weg zu einer, auch die sprachpragmatische Dimension einbeziehenden Historischen Semantik.

Die Begriffsgeschichte im herkömmlichen Format der Konzentration auf die Knotenpunkte im diachronen Bedeutungswandel einzelner Wörter, gestützt auf eine relativ schmale Basis von Belegzitaten aus Lexika, politischtheoretischen Texten und verstreuten anderen Quellen, die aus der Kommunikationssituation und Handlungsvollzügen weitgehend herausgelöst werden, wird trotzdem ihre Berechtigung behalten. Allerdings behält sie die Berechtigung vorzüglich im Sinne einer Hilfestellung zur Formulierung von Hypo-

26 Vgl. für eine Zusammenfassung des State of the Art auf diesem Gebiet: Bernhard Jussen: Ordo zwischen Ideengeschichte und Lexikometrie. Vorarbeiten an einem Hilfsmittel mediävistischer Begriffsgeschichte. In: Bernd Schneidmüller/Stefan Weinfurter (Hg.): Ordnungskonfigurationen im Hohen Mittelalter. Ostfildern 2006. S. 227-256.

27 Skeptisch: Gunter Scholtz: Vom Nutzen und Nachteil des Computers für die Begriffsgeschichte. In: Ernst Müller (Hg.): Begriffsgeschichte im Umbruch? Hamburg 2005. S. $185-194$.

Reinhart Koselleck: Hinweise auf die temporalen Strukturen begriffsgeschichtlichen Wandels (engl. 1994, dt. 2002). In: ders.: Begriffsgeschichten. Studien zur Semantik und Pragmatik der politischen und sozialen Sprache. Frankfurt/Main 2006. S. 86-98, hier S. 86; vgl. auch Kosellecks teils den lexikalischen Ansatz verteidigenden, teils seine Grenzen aufzeigenden Bemerkungen im letzten Textband des Lexikons: ders.: Vorwort. In: Otto Brunner/Werner Conze/Reinhart Koselleck (Hg.): Geschichtliche Grundbegriffe. Bd. 7. Stuttgart 1992. S. V-VIII. 
thesen zu umfassenderen Prozessen semantischen Wandels. So verstanden, als Subdisziplin einer Historischen Semantik, die sich zunehmend der Erforschung von größeren semantischen Feldern, Satzmustern, Diskursen oder languages im Sinne John Pococks widmet und dabei auch die sich wandelnden Gebrauchsformen und -situationen berücksichtigt, hat die Begriffsgeschichte alten Stils durchaus ihren Wert, denn es ist eine Methode, die relativ schnell zu überprüfbaren Ergebnissen führt. ${ }^{29}$,Historische Semantik‘ erscheint demgegenüber als geeignete übergeordnete Disziplinbezeichnung, um all diejenigen Forschungsrichtungen zu bündeln, die sich mit Prozessen des semantischen Wandels im weiteren Sinne befassen. Der Akzent kann dabei auf verschiedene Dimensionen semantischen Wandels gelegt werden. Historische Semantik, wie sie hier verstanden wird, umfasst demnach Forschungen zu Veränderungen sowohl im regelhaften Gebrauch sprachlicher (und anderer) Zeichen als auch in der Beziehung dieser Zeichen zu kognitiven Korrelaten (Begriffen) als auch in der Referenz dieser Zeichen auf außersprachliche Sachverhalte. ${ }^{30}$

\section{Eine offene Frage: Erklärungsmöglichkeiten für semantischen Wandel}

Ältere begriffsgeschichtliche ebenso wie neuere diskurshistorische Arbeiten haben ausgefeilte Instrumentarien entwickelt, um semantischen Wandel zu beschreiben; sie stoßen aber häufig an Grenzen, wenn es gilt, ihn zu erklären. Der Hauptgrund für dieses Problem scheint in beiden Fällen darin zu liegen, dass das sprachliche Handeln auf der Ebene der Mikrodiachronie vernachlässigt wird. Nur durch Einbeziehung der sprachpragmatischen Dimension und die Beobachtung sprachlicher Interaktion in kürzeren Zeit- und konkreten Handlungsräumen lassen sich - so die hier vertretene Auffassung - angemessene Modelle für die Erklärung semantischen Wandels entwickeln. Dass in diesem Punkt weder die Begriffsgeschichte noch die historische Diskursanalyse mit befriedigenden Ergebnissen aufwarten können, hängt mit ihren jeweiligen epistemologischen Grundannahmen und erkenntnisleitenden Interessen zusammen.

29 Einen sehr guten Überblick über verschiedene Forschungsansätze zwischen älterer Begriffsgeschichte und historischer Diskursanalyse vermittelt: Rolf Reichardt: Historische Semantik zwischen lexicométrie und New Cultural History. Einführende Bemerkungen zur Standortbestimmung. In: ders. (Hg.): Aufklärung und Historische Semantik. Interdisziplinäre Beiträge zur westeuropäischen Kulturgeschichte. (Zeitschrift für Historische Forschung, Beiheft 21). Berlin 1998. S. 7-28.

30 Ich stütze mich hier auf die Unterscheidung verschiedener Dimensionen von ,Bedeutung' bei: Ekkehard Felder: Semantische Kämpfe in Wissensdomänen. Eine Einführung in Benennungs-, Bedeutungs- und Sachverhaltsfixierungs-Konkurrenzen. In: ders. (Hg.): Semantische Kämpfe. Macht und Sprache in den Wissenschaften, Berlin/New York 2006. S. 13-46, hier bes. S. 20-33. 
Im Falle der Diskursanalyse im Anschluss an Michel Foucault resultieren die Schwierigkeiten bei der Konzeptualisierung von semantischem Wandel aus einer anhaltenden Scheu davor, strategisches Handeln von Subjekten einerseits, außersprachliche, durch historische Forschung (re)konstruierbare Konstellationen andererseits als Erklärungsmomente zuzulassen. Erkenntnistheoretisch liegt dieser Scheu die Annahme einer Unhintergehbarkeit der Sprache als Medium unserer eigenen und jeder vergangenen Wirklichkeitskonstitution zugrunde. Subjektive Intentionen (rationale Wahl) und außersprachliche Konstellationen werden in der Diskursanalyse ausgeklammert nicht weil man annimmt, dass es sie gar nicht gibt, sondern weil man sie nicht mit Gewissheit erkennen kann. ${ }^{31}$ Akzeptiert man diese gemeinsame Basis aller Spielarten des linguistic turn, bleiben als erklärende Kontexte für sprachliche Manifestationen der Vergangenheit nur andere überlieferte Sprachzeugnisse. Wenn strategisches Handeln von Subjekten wie auch außersprachliche Faktoren (beispielsweise die Positionierung von Subjekten im Raum oder die Verfügbarkeit bestimmter Medien) als Antriebsmomente beziehungsweise Anlässe semantischer Innovation ausscheiden sollen, liegt die gesamte Last der Erklärung auf innersprachlichen, anonymen, aus Sicht der Kommunikationsteilnehmer kontingenten Verschiebungen im Haushalt des jeweils Sagbaren. Warum-Fragen lassen sich dann kaum mehr sinnvoll stellen und noch weniger beantworten. Foucault selbst und die von ihm inspirierten Diskurshistoriker und -historikerinnen haben sich daher darauf konzentriert, derartige Verschiebungen des Sagbaren festzustellen. Ihnen ging es vorrangig um den Nachweis, dass sich - in Foucaults Terminologie - die Regeln des Erscheinens von Aussagen zwischen Zeitpunkt a und Zeitpunkt $b$ veränderten, weniger um die Analyse der Modalitäten und Gründe des Übergangs von a nach $b$. Verwiesen wird allenfalls auf in den Textgeflechten identifizierbare Brüche, Versprecher, Widersprüche, Interferenzen anderer Diskurse. Diese werden als Indizien dafür zitiert, dass individuellen Sprechern immer Spielräume blieben, Dinge anders zu sagen, als die Regeln es zuließen. ${ }^{32}$ Wie und warum sich aber einzelne Regelvariationen oder -durchbrechungen überindividuell und nachhaltig durchsetzten, andere hingegen nicht, bleibt weiterhin offen.

Reinhart Koselleck und die ihm folgenden Begriffshistoriker und -historikerinnen haben im allgemeinen weniger Hemmungen verspürt, zur Erklärung von semantischem Wandel auf eine ,Sachgeschichte “ jenseits der sprachlichen Überlieferung oder auf strategisches Handeln einzelner Sprecher zu verweisen. Es sind weniger prinzipielle epistemologische Bedenken als vielmehr

31 Vgl. Philipp Sarasin: Geschichtswissenschaft und Diskursanalyse. In: ders.: Geschichtswissenschaft und Diskursanalyse [wie Anm. 14]. S. 10-60, S. 29: „Dieses unschuldige, selbstverständliche Verstehen ist zweifellos genau das, womit Foucault brechen wollte, ...".

32 Ebd., S. 51-55. 
die Bevorzugung der diachronen Perspektive und das primär referentielle Verständnis von ,Bedeutung', die dazu geführt haben, dass in der Praxis des Lexikons „Geschichtliche Grundbegriffe“ semantischer Wandel eher beschrieben als erklärt wurde. Vorrangiges Erkenntnisziel der lexikographischen Begriffsgeschichte war es anfangs, die temporalen Verwerfungen zwischen dem Gebrauch bestimmter Wörter, den durch sie ausgedrückten Begriffen und einer mit anderen Methoden rekonstruierten ,Sachgeschichte politischer und sozialer Verhältnisse auszuleuchten. Das Hauptthema war - in der Diktion Kosellecks - die „Konvergenz" oder Divergenz zwischen der ,realen“ Geschichte einerseits und der von den Zeitgenossen ,begriffenen' Geschichte andererseits. ${ }^{33}$ Der Akzent lag auf der Identifizierung von Momenten, in denen bestimmte Wörter erstmals diejenigen abstrakten Bedeutungen annahmen beziehungsweise in sich aufnahmen, mit denen sie seit der ,Sattelzeit ${ }^{\text {‘ }}$ verbunden wurden. Die Begriffsgeschichten des Lexikons sollten vor allem zeigen, wann ältere Bedeutungen unverständlich wurden oder in Vergessenheit gerieten, wann sich die modernen, uns vertrauten Bedeutungen etablierten, schließlich ob sich dieser Bedeutungswandel im Einklang mit, im Nachgang zu oder im Vorgriff auf die ,reale' Geschichte vollzog. In diesem Sinne sprach Koselleck von „Bedeutungsüberhängen“, von „Vorgriffen“ auf zukünftige Geschichte, von verschiedenen Veränderungsgeschwindigkeiten des semantischen und des sozialen Wandels. ${ }^{34}$ Und so war anfangs wohl auch seine viel zitierte Formel gemeint, nach der begriffliche Veränderungen sowohl „Indikatoren" für sich wandelnde soziale, politische, ökonomische Verhältnisse, als auch „Faktoren“ im Wandel eben dieser Verhältnisse sein konnten. ${ }^{35}$ Wenn Koselleck von Begriffen als „Faktoren“ geschichtlicher Bewegung sprach, dachte er anfangs vor allem an den besonderen Fall des „Vorgriffs“ auf zukünftige Geschichte, das heißt an die vielen Begriffe, die seit dem Zeitalter der Aufklärung politisiert, ideologisiert, demokratisiert und verzeitlicht wurden und damit ein Erwartungspotential freisetzten, das die Vorstellungen und Handlungen der Menschen bestimmte. All diese frühen Formulierungen Kosellecks fassten Begriffsgeschichte einerseits, Real- oder Sozialgeschichte andererseits wie zwei weitgehend unverbundene Geschehensreihen auf. Wie und warum sich in der Interaktion zwischen sozialen Verhältnissen und Sprachgebrauch semantische Veränderungen vollzogen, wurde in den „Geschichtlichen Grundbegriffen“ kaum thematisiert. Aus dieser Fragerichtung erklärt sich, dass ,Bedeutung' im Lexikon primär im Sinne der Referenz auf außersprachliche Sachverhalte oder kognitive Korrelate (Begriffe) verstanden wurde, dagegen höchstens ausnahmsweise einmal im Sinne des regelhaften Gebrauchs im Kommunikationsfluss.

33 Reinhart Koselleck: Einleitung. In: Otto Brunner/Werner Conze/Reinhart Koselleck (Hg.): Geschichtliche Grundbegriffe. Bd. 1. Stuttgart 1972. S. XIII-XXVII, S. XXIII. 
Die in der Einleitung des Lexikons formulierten Erkenntnisziele lassen sich im Rückblick als Reaktion auf die disziplinäre Situation der Geschichtswissenschaft in der Entstehungszeit der Begriffsgeschichte verstehen. Die frühe Phase der Begriffsgeschichte, also die Zeit von den späten 1960er Jahren bis zur Mitte der 1980er Jahre, war zugleich die Zeit des Aufstiegs des struktur- beziehungsweise sozialgeschichtlichen Paradigmas in der Geschichtswissenschaft. Es ging den Sozialhistorikern - grob gesagt - um die Rekonstruktion gesellschaftlicher Makrostrukturen (zum Beispiel sozialer Klassen) und ihrer Kräfteverhältnisse in der Geschichte. Das geschah auf der Basis von zum Teil quantifizierend aufbereiteten Datenreihen, die aus verschiedenen Quellen erhoben wurden: Daten zu Einkommen, Steuerleistungen, Erbschaften, Heiratskreisen, Familiengrößen. Die historische ,Realität der so rekonstruierten Sozialstrukturen und Kollektivsubjekte war kein Diskussionsthema, ebenso wie die historische ,Realität' der auf ähnliche Weise rekonstruierten ökonomischen Konjunkturverläufe und der traditionell ermittelten politischen Ereignisgeschichte unstrittig war. Die heute geläufige konstruktivistische Sicht und die damit einhergehenden Zweifel an diesem unkritischen Verständnis historischer Wirklichkeit brachen erst seit Mitte der 1980er Jahre in die deutsche Geschichtswissenschaft ein. Auch in der Konzeptions- und frühen Entfaltungsphase der Begriffsgeschichte spielten Zweifel am Realitätscharakter der sozialhistorischen Rekonstruktionen noch kaum eine Rolle.

Seit dem Einbruch der linguistischen und konstruktivistischen Wende verlagerte sich jedoch bei Koselleck und denen, die in seinem Umkreis Begriffsgeschichte betrieben, das Interesse auf das ,Wie' und ,Warum' der sprachlichen Konstitution von Wirklichkeiten. ${ }^{36}$ Es wuchs das Bewusstsein dafür, dass soziale ,Strukturen' aller Art (Gruppen, Klassen, Gemeinschaften, Institutionen bis hin zu Staaten, Völkern, Nationen) sich im kommunikativen Vollzug, also durch Sprach- oder Symbolhandlungen, ständig neu hervorbringen (müssen) und sich dabei dauernd umkonfigurieren. ${ }^{37}$ Heute ist diese

36 Man vergleiche, um die unterschiedliche Akzentuierung zu sehen: Reinhart Koselleck: Begriffsgeschichte und Sozialgeschichte (1972). In: ders.: Vergangene Zukunft. Zur Semantik geschichtlicher Zeiten. Frankfurt/Main 1979. S. 107-129; ders.: Sozialgeschichte und Begriffsgeschichte (1986). In: ders.: Begriffsgeschichten [wie Anm. 28], S. 9-31. Wichtig auch Kosellecks Auseinandersetzung mit Gadamer: Reinhart Koselleck, Historik und Hermeneutik (1987). In: ders.: Zeitschichten. Studien zur Historik. Frankfurt/Main 2000. S. 97-118.

37 Vgl. Reinhart Koselleck: Sprachwandel und Ereignisgeschichte (1989). In: ders.: Begriffsgeschichten [wie Anm. 28]. S. 32-55; ders./Ulrike Spree/Willibald Steinmetz: Drei bürgerliche Welten? Zur vergleichenden Semantik der bürgerlichen Gesellschaft in Deutschland, England und Frankreich (1991). In: ebd., S. 402-461; Michael Jeismann: Das Vaterland der Feinde. Studien zum nationalen Feindbegriff und Selbstverständnis in Deutschland und Frankreich 1792-1918. Stuttgart 1992. Als Impuls für die Umorientierung war Dietrich Busses kritische Revision des begriffsgeschichtlichen Forschungsprogramms sehr wichtig: Dietrich Busse: Historische Semantik. Analyse eines Programms. Stuttgart 1987. 
Sicht in der Geschichtswissenschaft weitgehend common sense. Für die herkömmliche Sozial- beziehungsweise Gesellschaftsgeschichte, wie sie von Jürgen Kocka und Hans-Ulrich Wehler vertreten wurde, bedeutete sie aber zunächst eine erhebliche Provokation. Denn es stellte sich nun heraus, dass die vermeintlich stabilen Kollektivsubjekte, die bisher in den sozialgeschichtlichen Darstellungen als Subjekte figuriert hatten, im Grunde höchst instabile, ständig sich erneuernde, sprachlich hervorgebrachte Konstrukte waren. Für die herkömmliche Begriffsgeschichte brachte die neue Sicht eine Verschiebung des Fokus von der Langzeit-Diachronie auf die Mikro-Diachronie mit sich. Damit verbunden war eine Ablösung, mindestens aber eine Ergänzung des referentiellen Verständnisses von Bedeutung durch eines, das die Analyse der durch Sprechen und Verstehen (oder auch Missverstehen) immer neu hergestellten Bedeutung in den Mittelpunkt rückte. Im Rahmen des Lexikons „Geschichtliche Grundbegriffe“ und anderer lexikalischer Großprojekte konnte dieser Paradigmenwechsel schon aus darstellungstechnischen Gründen nur begrenzt mitvollzogen werden. ${ }^{38}$ Daher die erwähnte zunehmende Unzufriedenheit Kosellecks mit der ursprünglichen Konzeption der „Geschichtlichen Grundbegriffe". So notwendig für Hypothesenbildungen die auf lange Zeiträume bezogene diachrone Begriffsgeschichte im Lexikonformat bleibt, so wenig ist sie geeignet, die ständige Verfertigung sozialer, politischer, rechtlicher, ökonomischer und sonstiger Strukturen und Verhältnisse durch sprachliches Handeln zu erklären und - umgekehrt - Erklärungen dafür anzubieten, warum unter gegebenen Umständen die Akteure so sprachen, wie sie sprachen.

Konkrete Vorgänge des semantischen Wandels, der Bedeutungsanreicherung, -verschiebung oder -verdrängung lassen sich besser in der MikroDiachronie verfolgen. Nur durch die Betrachtung sprachlicher Interaktionen in Handlungssituationen wird erkennbar, wie und warum Sprecher(innen)/ Schreiber(innen) bestimmte Wörter einmal so, ein anderes mal anders verwendeten, und wie und warum Hörer(innen)/Leser(innen) sie so oder anders verstanden. Ein Problem, das sich aus der Verengung der Linse auf die MikroDiachronie ergibt, besteht nun darin, dass die Verkürzung der Untersuchungszeiträume und die Vermehrung der zu analysierenden Textsorten zu größerer Unübersichtlichkeit führt. Die großen, linearen Erzählungen der älteren Begriffsgeschichte lösen sich tendenziell auf in viele kleine Geschichten situationsbedingten Sprachgebrauchs, die keinen allgemeinen Verlaufsmustern mehr zu folgen scheinen.

Lassen sich dennoch wiederkehrende (oder gar zeittypische) Muster der Interaktion zwischen semantischem Wandel und sozialem Wandel identifizie-

38 Vgl. aber den - nicht zufällig zu Monographiegröße angewachsenen - Artikel zu ,Volk, Nation": Fritz Gschnitzer/Reinhart Koselleck/Bernd Schönemann/Karl Ferdinand Werner: Volk, Nation, Nationalismus, Masse. In: Otto Brunner/Werner Conze/Reinhart Koselleck (Hg.): Geschichtliche Grundbegriffe. Bd. 7. Stuttgart 1992. S. 141-431. 
ren? Dies ist eine offene Frage, zu der hier vorläufig nur abstrakte Hypothesen angeboten werden können. Drei typische Verlaufsformen kommen für die Erklärung semantischen Wandels in Frage: (1) Wandel durch Plausibilitätsverlust von Wörtern oder Redeweisen infolge überraschender Ereignisse und Umbrüche; (2) Wandel durch $\mathrm{Zu}$ - oder Abnahme des strategischen Gebrauchswerts von Wörtern oder Redeweisen in wiederkehrenden Kommunikationssituationen; (3) Wandel durch Irritation des Wort- und Bedeutungshaushalts einer Sprache durch Wortimporte aus einer anderen Sprache.

Das erste Erklärungsmodell - Wandel durch Plausibilitätsverlust bisheriger Redeweisen - lässt sich an vielen Beispielen in der Geschichte belegen. Der klassische Fall sind plötzlich eintretende, ,unerhörte‘ Ereignisse, die nicht mehr mit bisher gemachten Erfahrungen vereinbar sind. Das zur Verfügung stehende Vokabular reicht nicht mehr aus, um das ,Unerhörte' des Neuen begrifflich zu fassen. Der Frühneuzeithistoriker Andreas Suter hat diesen Vorgang an einem schönem Beispiel, den sprachlichen Reaktionen der Beteiligten auf den Schweizer Bauernkrieg von 1653, verdeutlicht. Als die Bauernaufstände begannen, reagierten die Obrigkeiten in den Schweizer Kantonen gelassen; sie meinten, es handele sich um eine der üblichen „Unruhen“, „Revolten“ oder „Widersetzlichkeiten“, die ihnen vertraut waren und für deren Niederschlagung sie Routinen entwickelt hatten. Die Ausweitung des Aufstands auf weite Teile der Schweiz jagte den Obrigkeiten jedoch panischen Schrecken ein; sie sahen sich nun „verfahrungen und extremitäten“ gegenüber, „die wir uns nyt hetten ynbilden können“. ${ }^{39}$ Und genau in dieser Situation, in der etwas geschah, was sie sich vorher ,nyt hetten ynbilden können“, begannen die Obrigkeiten nun, die ,Unruhen' anders zu bezeichnen, nämlich als „Generalverschwörung“ oder - und das war sogar im europäischen Rahmen eine begriffliche Innovation - als „Revolution". ${ }^{40}$ Suter zeigt weiter, dass im Laufe der verschiedenen Schweizer Aufstände auch seitens der revoltierenden Bauern neue sprachliche und symbolische Formen, ihren Forderungen Ausdruck zu verleihen, gefunden wurden.$^{41}$ Erklären lassen sich diese Vorgänge der Umsemantisierung, so Suter, durch Erfahrungen des Scheiterns, präziser: durch die Erfahrung ,der Falsifizierung der semantischen Systeme und Begriffe, sei es, weil der Akt der Zuschreibung nicht mehr gelingt, sei es, weil die aus derartigen Zuschreibungsakten abgeleiteten Handlungsweisen nicht mehr die erwarteten Ergebnisse zeitigen. “42 Dies als Beispiel für semantischen Wandel durch Plausibilitätsverlust. Verallgemeinert und extrem vereinfacht gesagt: Es geschieht etwas Unvorhergesehenes; man

39 Andreas Suter: Kulturgeschichte des Politischen - Chancen und Grenzen. In: Barbara Stollberg-Rilinger $(\mathrm{Hg}$.$) : Was heißt Kulturgeschichte des Politischen? (Zeitschrift für$ Historische Forschung, Beiheft 35). Berlin 2005. S. 27-55, S. 33.

40

Ebd., S. $31 \mathrm{f}$.

Ebd., S. $44 \mathrm{ff}$.

Ebd., S. 34. 
stellt fest, dass bisherige Redeweisen weder den neuen Sachverhalt erfassen noch erfolgreiches Handeln in der neu entstandenen Situation erlauben; und man verändert daraufhin seine Redeweisen.

Was freilich mit diesem Verlaufsmodell noch nicht hinreichend erklärt werden kann, ist die konkrete neue Wortwahl, die an die Stelle der alten tritt. Warum wurde ausgerechnet das Wort „Revolution“ und nicht irgendein anderes verwendet, um die Neuartigkeit der Erfahrung im Schweizer Bauernkrieg auf den Begriff zu bringen? Damit bin ich beim zweiten der zuvor genannten (und komplementären) Erklärungsmodelle. Plausibilitätsverlust ist eine mögliche Erklärung für den Rückgang oder die Verdrängung bisheriger Redeweisen ${ }^{43}$, erklärt aber für sich allein noch nicht das Auftauchen und die Ausbreitung spezifischer neuer Redeweisen.

Hier greift nun das zweite Verlaufsmodell, semantischer Wandel durch Zunahme des strategischen Gebrauchswerts in der Kommunikationssituation. Extrem vereinfacht gesagt, läuft es darauf hinaus, dass bestimmte Wörter sich im Gebrauch durchsetzen, weil sich mit ihnen erfolgreich sprechen und handeln lässt - was immer ,Erfolg' im Einzelfall sein mag; das ist situationsabhängig. Worin nun der Mehrwert des Worts ,Revolution' gegenüber den älteren Bezeichnungen ,Unruhe', ,Revolte' usw. in der Situation des Schweizer Bauernkriegs bestand, vermag ich mangels empirischer Sachkenntnis nicht mit Sicherheit zu sagen. Allgemein lässt sich vermuten, dass der höhere Grad an Abstraktion, der im Bild der revolutio enthalten war, ein Grund für die Wahl gerade dieses (metaphorischen) Ausdrucks war. Noch allgemeiner: Wörter gewinnen in der politischen Kommunikation an Gebrauchswert, wenn sie die Fähigkeit besitzen, möglichst viele disparate Einzelerfahrungen $\mathrm{zu}$ bündeln. In der politischen Kommunikation sind möglichst abstrakte, nach vielen Seiten hin anschlussfähige und vielseitig auffüllbare Ausdrücke besonders vorteilhaft. Sofern man als politischer Akteur auf die - periodisch zu bekräftigende - Zustimmung anderer angewiesen ist, hat man ein Interesse daran, Wörter zu verwenden, die mehrere Optionen offen lassen. Günstig sind Redeweisen, die es gestatten, unhaltbare Positionen unauffällig zu räumen, ohne das Vokabular ändern zu müssen. Von Vorteil sind auch Kollektivbezeichnungen, die breite Aktionsbündnisse ermöglichen, also möglichst inkludierend sind, oder umgekehrt: Bezeichnungen, die das ganz Andere, zu Exklu-

43 Eine andere, hier nicht näher betrachtete Erklärung für den Rückgang oder gar das Verschwinden von Wörtern aus dem Gebrauch kann die Überfrachtung mit widersprüchlichen Bedeutungen sein, die dazu führt, dass ihr Gebrauch in bestimmten Situationen dysfunktional wird. Man könnte von Bedeutungsverlust durch zu weit getriebene Polysemie sprechen. Ein Beispiel ist die zunehmende Unbrauchbarkeit der Bezeichnung ,Bürger" in den parlamentarischen Diskussionen um Bürgerrechte in den Landtagen des Vormärz und in der Paulskirche; vgl.: Willibald Steinmetz: „Sprechen ist eine Tat bei euch." Die Wörter und das Handeln in der Revolution von 1848. In: Dieter Dowe/Heinz-Gerhard Haupt/Dieter Langewiesche (Hg.): Europa 1848. Revolution und Reform. Bonn 1998. S. 1089-1138, hier S. 1095 ff. 
dierende möglichst inhaltsleer lassen. All das erklärt die hohe Attraktivität abstrakter, im Extremfall sogar , leerer ' Signifikanten in der Politik. ${ }^{44}$ Rekurse auf Intentionalität sind nicht unbedingt erforderlich, um den Gebrauch strategisch günstiger Vokablen zu erklären; ich sehe allerdings auch keinen Grund, warum man sie als Erklärung in den Fällen ausschließen sollte, in denen sie durch explizite Hinweise in Parallelquellen eindeutig als solche ansichtig werden.

Ein Beispiel für semantischen Wandel durch Zunahme strategischen Gebrauchswerts ist die Verwendung bestimmter sozialer Beschreibungsbegriffe in parlamentarischen Diskussionen über Wahlrechtsreformen des 19. Jahrhunderts. ${ }^{45}$ Die bemerkenswerte Konjunktur der Bezeichnungen ,classes moyennes ${ }^{6}$ in den französischen beziehungsweise ,middle classes ${ }^{6}$ in den englischen Wahlrechtsdebatten der ersten Hälfte des 19. Jahrhunderts lässt sich auf diese Weise erklären. Wer im Parlament an die ,classes moyennes' oder die ,middle classes' appellierte, wer behauptete, die ,Mittelklassen' seien auf seiner Seite, signalisierte damit, dass die eigene Position breite Unterstützung in der Bevölkerung fand. Die Ausdrücke ,middle classes" oder ,classes moyennes' ließen es aber offen, welche konkreten Berufs- und Interessengruppen man meinte. Und genau diese Bedeutungsoffenheit von Wörtern (im referentiellen Sinne) war - und ist - in der politischen Kommunikation von Vorteil. In nicht-politischen Kommunikationssituationen, beispielsweise in der juristischen Kommunikation vor Gericht, ist eher das Gegenteil der Fall. Wer dort nicht in der Lage ist, seinen persönlichen Fall in die präzise Fachterminologie zu übersetzen, die dem Richter die juristische Entscheidung erlaubt, wird Nachteile erleiden. Es lassen sich somit keine generellen, sondern nur situationsspezifische Regeln für den Gebrauchswert von Wörtern und Redeweisen angeben. Und selbstverständlich variieren diese situationsspezifischen Regeln auch historisch in dem Maße, wie sich die institutionalisierten oder routinisierten Formen der Kommunikation selbst, also zum Beispiel Wahlkämpfe, parlamentarische Verfahren, Gerichtsverhandlungen, ändern.

Die beiden bisher erläuterten Erklärungsmodelle für semantischen Wandel, Änderung der Wortwahl aufgrund Plausibilitätsverlusts und Zunahme des Gebrauchswerts in der Situation, basieren darauf, dass Sprecher Erfahrungen machen und Erwartungen hegen, für die sie - zielstrebig oder tastend im trial and error-Verfahren - nach geeigneten sprachlichen Ausdrucksweisen suchen. Beides sind Erklärungsmodelle, die Akteure mit bestimmten identifizierbaren Interessen voraussetzen. Diese Voraussetzung muss keineswegs so weit gehen,

44 Vgl. Ernesto Laclau: Why do Empty Signifiers Matter to Politics? In: Jeffrey Weeks (Hg.): The Lesser Evil and the Greater Good. London 1994. S. 167-178.

45 Vgl. zum folgenden: Willibald Steinmetz: Gemeineuropäische Tradition und nationale Besonderheiten im Begriff der ,Mittelklasse'. Ein Vergleich zwischen Deutschland, Frankreich und England. In: Koselleck/Schreiner (Hg.): Bürgerschaft [wie Anm. 23], S. 161-236, hier S. 211-233. 
dass man den Akteuren ein instrumentelles Sprachverhalten unterstellt. Gezielte Suche nach der in der Situation ,optimalen' Wortwahl oder gezielte Tabuisierung eines Wortgebrauchs dürften die Ausnahme sein. ${ }^{46}$ Der Normalfall ist die unbewusste Steuerung der Wortwahl durch kleine Erfolgs- oder Misserfolgserlebnisse im alltäglichen Kommunikationsfluss. Schwierig wird es allerdings, wenn man semantischen Wandel erklären will, ohne dabei auf Akteure und ihr Streben nach gelingenden Sprachhandlungen zu rekurrieren. Ich kann mir vorläufig nur einen Modus semantischen Wandels vorstellen, der ohne die Unterstellung eines Strebens nach gelingenden Sprachhandlungen auskommt. Es ist das dritte genannte Erklärungsmodell: semantischer Wandel durch Irritation infolge von Wortimporten von der einen in die andere Sprache.

Wie erwähnt, war Mehrsprachigkeit in vielen europäischen Territorien bis ins 19. Jahrhundert hinein der Normalfall. Es kam daher häufig vor, und flüchtige Blicke in frühneuzeitliche Schriftquellen jedweder Art bestätigen es, dass Wörter aus einer Fremdsprache (Latein, Französisch) als ,fremde ${ }^{6}$ Wörter mit zunächst oft diffuser Bedeutung Eingang in die eigene (gesprochene und geschriebene) Sprache fanden. Auf welchen Transferwegen, durch welche Medien vermittelt und von wem ausgehend diese Wortimporte und -exporte jeweils geschahen, ist ein eigenes historisch-semantisches Forschungsfeld, auf dem bisher wenig geschehen ist, wenn man vom Teilgebiet der historischen Übersetzungsforschung absieht. ${ }^{47}$ Tatsache ist, dass Wortimporte aus Fremdsprachen Initialzündungen für semantische Verschiebungen sein können. Ähnliche Irritationen können durch Wortimporte aus Fachsprachen ausgelöst werden, man denke nur an die Computerterminologie. Die fremden, in ihrer Bedeutung zunächst nur vage fixierten Wörter, sind gewissermaßen Spielmaterial, das sich zu vielfältigen Zwecken nutzen lässt. So kann man beispielsweise auf ein Fremdwort bestimmte negative Konnotationen eines bedeutungsverwandten Worts der eigenen Sprache abwälzen und damit eine vorher so nicht mögliche semantische Differenzierung herbeiführen. Ein klassisches Beispiel ist die Negativ-Konnotation des französischen Worts ,bourgeois' im Deutschen spätestens seit Marx, wodurch das deutsche Wort ,Bürger' auch ohne positive adjektivische oder substantivische Zusatzbestimmungen einen positiven Klang behielt. Ein anderes, von Ingrid Schierle und Walter Sperling nachgezeichnetes Beispiel ist die Einführung des

46 Forderungen nach ,politisch korrektem' Sprachgebrauch fallen in die letztgenannte Kategorie.

47 In einem glänzenden Überblick behandelt von: László Kontler: Translation and comparison - translation as comparison. www.eui.eu/HEC/Research Teaching/ 20052006-Spring/ss-reading-kontler.pdf (Zugriff am 13. April 2007); ergänzend: Doris Bachmann-Medick: Einleitung: Übersetzung als Repräsentation fremder Kulturen. In: dies. (Hg.): Übersetzung als Repräsentation fremder Kulturen. Berlin 1997. S. 1-18; Douglas Howland: The Predicament of Ideas in Culture: Translation and Historiography. In: History and Theory 42 (2003), S. 45-60. 
Fremdworts ,Politik' (,politika') ins Russische. Schierle und Sperling zeigen, wie sich das Politikvokabular im Russischen nach seiner Einführung durch Übersetzungen zunächst ausdifferenzierte, um dann im Zuge der Partizipationskämpfe im späten Zarenreich von einem zunächst pejorisierten Randbegriff zum umstrittenen Kampfbegriff zu werden. ${ }^{48}$ Ein drittes Beispiel, von Koselleck angeführt, ist die Luthersche Übersetzung des biblischen Wortes ,berith' durch das deutsche Wort ,Bund'. Durch diesen Übersetzungsvorgang erhielt der bis dahin rein weltliche Terminus ,Bund“ eine religiöse Aufladung, die bis ins 19. Jahrhundert hinein abrufbar blieb ${ }^{49}$ Neu eingeführte Fremdwörter erlauben also semantische Differenzierungsprozesse. Auch das vollzieht sich freilich nicht quasi-autonom im System der Sprache. Sind die Fremdwörter einmal importiert, spielt auch hier das Streben benennbarer Akteure nach gelingenden Sprach handlungen eine entscheidende Rolle. Insofern verhält sich das dritte Erklärungsmodell semantischen Wandels komplementär zu den beiden anderen.

\section{Begriffsgeschichte transnational: Übersetzungsvorgänge und das Problem der Übersetzbarkeit von Begriffen}

Die eingangs erwähnten Projekte einer vergleichenden oder beziehungsgeschichtlichen ${ }^{50}$ historischen Semantik befassen sich mit Gesellschaften, in denen eine nach funktionalen, sozialen, ethnischen, religiösen oder sonstigen Kriterien differenzierte Mehrsprachigkeit herrschte. Es war eine Mehrsprachigkeit, die zudem im Falle der kolonialen Gesellschaften mit einem erheblichen Machtgefälle zwischen Sprechern einher ging. So war etwa im mittelalterlichen Iran seit dem Beginn der von türkischen Kriegern gegründeten Dynastien „Türkisch die Sprache der Herrscher und des Militärs, Persisch dagegen die Verwaltungs- und Kultursprache und Arabisch die Sprache von Religion und Recht.“ ${ }^{51}$ Die außersprachlich fundierte, jedoch mit sprachlichen Mitteln aufrechterhaltene Macht privilegierter Kommunikationsteilnehmer und ,ihrer' Sprache über andere ist aber auch in nicht-kolonialen Gesellschaften typisch für Situationen der Mehrsprachigkeit auf einem Territorium. Die Beachtung der jeweiligen Machtverhältnisse muss daher Teil jeder beziehungsgeschichtlichen historisch-semantischen Studie sein. ${ }^{52}$

48 Ingrid Schierle: Semantiken des Politischen im Russland des 18. Jahrhunderts; Walter Sperling: Vom Randbegriff zum Kampfbegriff: Semantiken des Politischen im ausgehenden Zarenreich (1850-1917). Beide in: Steinmetz (Hg.): ,Politik' [wie Anm. 11], S. 226-247 und 248-288.

49 Vgl. Koselleck, Hinweise auf die temporalen Strukturen [wie Anm. 28], S. 95 f.

so Zum Ansatz der Beziehungsgeschichte (histoire croisée) in der Geschichtswissenschaft allgemein: Michael Werner/Bénédicte Zimmermann: Beyond Comparison: Histoire Croisée and the Challenge of Reflexivity. In: History and Theory 45 (2006), S. 30-50.

s1 Albert Hourani: Die Geschichte der Arabischen Völker. Frankfurt/Main 1992. S. 123.

52 In einer gegenwartsbezogenen, normativ-politikwissenschaftlichen Perspektive wird diese Sprach-Machtfrage unter dem Leitbegriff „Language rights“ diskutiert. Vgl. im 
Es gibt allerdings keine allgemeine Regel, nach der etwa politische, militärische, wirtschaftliche Dominanz einer bestimmten Bevölkerungsgruppe oder die Herrschaft von Eroberern/Kolonisierern notwendigerweise zur Dominanz ihrer Sprache über die Sprache der unterlegenen Bevölkerungsgruppe, der Eroberten/Kolonisierten führen muss. Zwar lassen sich zahlreiche Beispiele dafür anführen, dass die Sprachen der europäischen Kolonialmächte vom 16. zum 20. Jahrhundert einen massiven Veränderungsdruck auf Sprachen kolonisierter Bevölkerungen oder semi-kolonialer Mächte (Indien, China, Japan) ausgeübt haben und dort zu Lehnübersetzungen oder Umsemantisierungen einheimischer Termini bis hin zu Übernahmen ganzer institutioneller Wert- und Normsysteme geführt haben; die in längeren Prozessen modifizierender Aneignung vollzogenen Transfers westlicher Begriffe, darunter auch der Kategorien des deutschen Bürgerlichen Gesetzbuchs nach Japan sind ein eindrückliches Beispiel ${ }^{53}$, die Fixierung des ursprünglich europäischen (portugiesischen) Terminus ,Kaste“ im Indischen des 19. Jahrhunderts ein anderes. ${ }^{54}$ Aber historisch gesehen gibt es auch den umgekehrten Fall: einen Veränderungsdruck auf die Sprache der Eroberer - bis hin zu deren völliger Transformation. Das klassische Beispiel ist die sprachliche Romanisierung, verbunden mit der religiösen Christianisierung, der germanischen Eroberer des Weströmischen Reiches. Der Normalfall dürfte eine lang andauernde Interferenz der Sprachen und Hybridität der Sprecher sein. Hinund hertransferiert werden dabei hauptsächlich nominale Elemente, und hier besonders Kulturwörter. ${ }^{55}$

Jenseits empirischer Studien zu Transfer- und Übersetzungsvorgängen stellt sich die grundsätzliche Frage nach der Übersetzbarkeit von Begriffen. Zu unterscheiden sind hier drei Teilprobleme, (1) das Problem der Äquivalenz auf der synchron-vergleichenden Ebene, (2) das Problem der Äquivalenz in der Diachronie, (3) das Problem des Eurozentrismus beziehungsweise der Modernelastigkeit der Metasprache, die wir benötigen, um uns über Äquivalenz und Übersetzbarkeit verständigen zu können.

Das erste Problem, die Frage der Äquivalenz (im Sinne der Gebrauchsweisen wie im referentiellen Sinne) auf der synchron-vergleichenden Ebene,

Überblick: Alan Patten/Will Kymlicka: Introduction: Language Rights and Political Theory: Context, Issues, and Approaches. In: dies. (Hg.): Language Rights and Political Theory. Oxford 2003. S. 1-51.

53 Hierzu: Shingo Shimada: Die Erfindung Japans. Kulturelle Wechselwirkung und nationale Identitätskonstruktion. Frankfurt/New York 2000; Douglas Howland: Translating the West: Language and Political Reason in Nineteenth-Century Japan. Honolulu 2002; ders.: Personal Liberty and Public Good: The Introduction of John Stuart Mill to Japan and China. Toronto 2005.

54 Vgl. Martin Fuchs: Kampf um Differenz. Repräsentation, Subjektivität und soziale Bewegungen. Das Beispiel Indien. Frankfurt/Main 1999. S. 52-54.

55 Vgl. Harald Weinrich: Mit gemischten Sprachgefühlen. In: ders.: Wege der Sprachkultur. München 1988. S. 290-306, hier S. 292 u. 294. 
wird in der europäischen Moderne vor allem bei Begriffen virulent, die in hohem Maße mit einer spezifischen nationalen Kultur und entsprechenden Institutionen verbunden sind. So lässt sich fragen, ob es für die schon von den Zeitgenossen als spezifisch ,deutsch“ wahrgenommenen Begriffe ,Bildung', ,Staat', , bürgerliche Gesellschaft', ,Kultur überhaupt jeweils zeitgenössische Begriffe in anderen europäischen Sprachen gab, die zumindest halbwegs ähnliche Bedeutungsspektren und Gebrauchsformen aufwiesen. Dieses synchrone Äquivalenzproblem ist für vergleichende historisch-semantische, aber auch sozial-, kultur- und politikhistorische Untersuchungen zentral. Verneint man die Frage der Äquivalenz, sind die betreffenden Begriffe als analytische Kategorien für Vergleiche nur mit größerem definitorischen Aufwand verwendbar. Ein möglicher Weg, die Frage zu beantworten, ist die Beobachtung vergangener Übersetzungsversuche. Man wird dabei aber vielfach nur auf eine Geschichte von Missverständnissen, neutraler gesagt: Umdeutungen und Anpassungen an andere kulturelle und institutionelle Kontexte stoßen. So übersetzten beispielsweise, wie Fania Oz-Salzberger zeigt, deutsche Rezipienten der schottischen Moralphilosophie des 18. Jahrhunderts die englischen Termini „community“, ,polity“ oder „nation“ häufig mit „Staat“, wodurch ihre klassisch-republikanische Bedeutung, die sie insbesondere bei Adam Ferguson besaßen, nicht nur verloren ging, sondern geradezu in eine obrigkeitsfreundliche verkehrt wurde. ${ }^{56}$ Geringer, aber immer noch beachtlich, sind die Bedeutungsdifferenzen, wenn es sich um Termini handelt, deren Wortstamm der gemeineuropäischen lateinischen Tradition entstammt - so etwa im Fall der Partei- und Ideologiebezeichnung ,Liberalismus ${ }^{6}{ }^{57}$ Die Untersuchung zeitgleicher oder zeitverschobener Übersetzungsversuche bietet mithin keine sichere Richtschnur, um die Frage der Äquivalenz zu lösen.

Eine Alternative könnten onomasiologische Studien bieten. Sie wären in der Weise anzulegen, dass ergebnisoffene Testfragen an Texte aus vergleichbaren vergangenen Kommunikationssituationen, zum Beispiel Debatten um Wahlrechte und politische Partizipation, gerichtet werden. Auf diesem Weg könnte es gelingen, zentrale Termini mit ähnlichem strategischen Gebrauchswert, also mit äquivalenter ,Bedeutung' im sprachhandlungstheoretischen Sinne, überhaupt erst aufzufinden. Bezogen auf Wahlrechtsdebatten könnte man also fragen, wie sich in einem bestimmten Zeitraum jeweils diejenigen sozialen Gruppen nannten, die Forderungen nach Anerkennung, Mitsprache, Inklusion aufstellten. Ebenso könnte man nach Fremdbezeichnungen für die Gruppen suchen, die Anerkennung, Mitsprache, Inklusion begehrten. Weiter könnte man die konkurrierenden Benennungen für die angestrebte Form des Mitreden-Wollens sowie die Legitimitationsformeln untersuchen, mit Hilfe

56 Vgl. Fania Oz-Salzberger: Translating the Enlightenment. Scottish Civic Discourse in Eighteenth-Century Germany. Oxford 1995. S. 46 u. 138-166.

57 Hierzu die Studie von Jörn Leonhard: Liberalismus. Zur historischen Semantik eines europäischen Deutungsmusters. München 2001. 
derer die Forderungen untermauert oder zurückgewiesen wurden. Damit dieses onomasiologische Verfahren praktikabel ist, müssen allerdings zwei Voraussetzungen vorliegen: Es muss vergleichbare und etwa gleichzeitige Kommunikationssituationen geben. Und man muss für die Formulierung der Testfragen über Kategorien verfügen, deren kultur- und nationsübergeifende Geltung angenommen werden kann. Beide Voraussetzungen sind für eine Geschichtsschreibung, die im west- und mitteleuropäischen Rahmen verbleibt, leichter zu erfüllen als für eine globale Geschichtsschreibung.

Das zweite Problem - Äquivalenz in der Diachronie - soll hier nur kurz gestreift werden, denn es besitzt für Ethnologen, Soziologen und Linguisten, sofern sie sich primär mit fremden oder eigenen Gegenwartsgesellschaften und ihrer Sprache in kurzen Zeiträumen befassen, nur eine geringere Relevanz. In der Geschichtswissenschaft ist es hingegen allenthalben präsent. Forschungspraktisch und darstellungstechnisch taucht das diachrone Übersetzungsproblem vor allem auf, wenn es um die Frage geht, ob moderne Begriffe zugleich als analytische Kategorien der historischen Darstellung zu früheren Epochen dienen können. Der klassische, in der Mediävistik bis heute kontrovers diskutierte Fall ist die Frage, ob man bezogen auf das Früh- und Hochmittelalter von ,Staaten', ,Verfassungen' oder zumindest ,Staatlichkeit' reden sollte. Das diachrone Äquivalenzproblem, genauer: die Gefahr der Verwendung anachronistischer analytischer Begrifflichkeiten, war bekanntlich ein wesentlicher Anstoß für die Entwicklung der Begriffsgeschichte, insbesondere bei Otto Brunner. ${ }^{58}$ Gute Begriffsgeschichten für die jeweils betrachteten Länder und Sprachräume sind daher eine notwendige Voraussetzung, um mit vergleichenden oder transfergeschichtlichen historisch-semantischen Studien überhaupt beginnen zu können.

Das dritte genannte Problem - die Gefahr des Eurozentrismus beziehungsweise der Modernelastigkeit der „Metasprache“ (Koselleck) wissenschaftlicher Verständigung - nimmt an Bedeutung zu, je mehr die Historische Semantik als Disziplin den europäisch-westlichen Raum verlässt und sich globalhistorisch ausweitet. ${ }^{59}$ Zugespitzt lässt sich fragen, ob die Eurozentrismus-Falle überhaupt vermeidbar ist. Kann es ,neutrale' analytische Termini geben, die historische oder kulturwissenschaftliche Darstellungen strukturieren, ohne die ,fremde' Kultur an eigenen Begriffen zu messen? Sollte man, um der Gefahr zu entgehen, nicht lieber auf zeitgenössische Quellenbegriffe der jeweils untersuchten fremden Kultur zurückgreifen? Die Südasien-Historikerin Margrit Pernau hat dieses Problem am Beispiel der Frage erörtert, ob

58 Vgl. dazu die Bemerkungen bei: Reinhart Koselleck: Begriffsgeschichtliche Probleme der Verfassungsgeschichtsschreibung. In: ders.: Begriffsgeschichten [wie Anm. 28], S. $365-401$.

59 Zum Problem der Nicht-Verfügbarkeit einer „Metasprache“ für vergleichende historisch-semantische Untersuchungen: Koselleck/Spree/Steinmetz: Drei bürgerliche Welten? [wie Anm. 37]. S. 413. 
man bezogen auf Indien unter britischer Herrschaft von einem indo-muslimischen ,Bürgertum' oder einer ,Mittelklasse' sowie einer ,Zivilgesellschaft" sprechen könne. ${ }^{60}$ Sie kommt zum Schluss, dass die Verwendung nicht-westlicher, quellensprachlicher Termini nicht nur das Verständnis behindere, sondern eine grundlegende, essentielle Andersartigkeit der indigenen Kulturen unterstelle und damit ebenfalls als eurozentrisch anzusehen sei. Pernau sieht daher in Darstellungen für ein westliches Lesepublikum keinen anderen Ausweg als den, die den westlichen Lesern und Leserinnen vertrauten Termini zu benutzen, diese allerdings durch genaue Darlegung der Bedeutungsdifferenzen gleichsam semantisch anzureichern, so dass man sich nach der Lektüre unter einem ,Bürger' auch einen indischen ,sharif ' mit Turban vorstellen könne. Folgte man diesem Rat, erhielten Studien zur vergleichenden und beziehungsgeschichtlichen Historischen Semantik ein Stück weit auch den Charakter von Begriffspolitik - bezogen auf die Wissenschaftssprache, wenn nicht sogar die Umgangssprache.

So einleuchtend der Grundgedanke, die Unvermeidbarkeit westlicher analytischer Termini, ist, bliebe gleichwohl sorgfältig zu überlegen, welche westlichen (deutschen, englischen) Termini man zum Objekt derartiger semantischer Anreicherungsoperationen macht. Es erscheint mir zumindest problematisch, wenn man ohnehin schon mit Bedeutungen überladene und bereits innerhalb Europas kaum adäquat übersetzbare Termini wie das deutsche Wort ,Bürger' auswählt, um sie sich für die Untersuchung nichteuropäischer Verhältnisse gefügig zu machen. Weniger kulturspezifische Bezeichnungen wie ,Mittelklasse ${ }^{\text {‘ }}$ oder ,Zivilgesellschaft ${ }^{`}$ mögen für diesen Zweck besser geeignet sein, wenngleich im Fall des normativ aufgeladenen Begriffs ,Zivilgesellschaft" wiederum in besonderem Maße die Gefahr droht, die Geschichte nicht-westlicher Länder primär unter dem Gesichtspunkt eines Defizits zu schreiben. Dieses Problem einer Historiographie im Modus des Erfolgs oder Misserfolgs bei der Annäherung an ein durch normative Begriffe definiertes Ideal stellt sich im übrigen genauso auch innerhalb Europas. ${ }^{61}$ Die gesamte historische Diskussion über deutsche und andere ,Sonderwege $^{6}$ ist letztlich ein Ausfluss der Schwierigkeit, Analysekategorien zu wählen, die Differenz, Andersartigkeit, andere Veränderungsrhythmen erkennen lassen, ohne diese als ,unnormal', ,abweichend', ,rückständig' zu charakterisieren. Mit Blick auf nicht-europäische Gesellschaften erhält das Problem jedoch zusätzliche Brisanz, betrifft es doch hier selbst Fundamentalkategorien wie Raum- und Zeitvorstellungen, oder vermeintliche Universalien wie ,Religion', ,Individuum', ,Politik', deren Existenz und ungefähre Bedeutung zumindest in der modernen europäischen Geschichte nicht in Frage steht. Das zu großen Teilen aus der gemeinsamen griechisch-lateini-

$60 \mathrm{Vgl}$. die oben in Anm. 13 genannten Publikationen.

61 Darauf weist auch Leonhard, Liberalismus [wie Anm. 56], S. 71, hin. 
schen Tradition stammende abstrakte Vokabular in Wissenschaft, Recht und vielen anderen Praxisbereichen übte in der europäischen Geschichte gewissermaßen einen Vereinheitlichungssog aus, für den es in der älteren, vorkolonialen Geschichte der nicht-westlichen Länder, vielleicht mit Ausnahme des ostasiatischen Raums (China, Japan) kein Äquivalent gegeben hat. ${ }^{62}$ Seit dem Beginn der Kolonialisierung sind jedoch auch die Sprachen kolonialer und semi-kolonialer Gesellschaften in diesen Vereinheitlichungssog hineingezogen worden. Durch westliche Sprachen vermittelte Begriffe griechisch-lateinischer sowie - in jüngerer Zeit - anglo-amerikanischer Herkunft bilden seit dem 19. Jahrhundert gleichsam einen Firnis an gemeinsamem Vokabular, der das Problem der Übersetzbarkeit langfristig geringer werden lassen könnte.

62 Vgl. die Bemerkungen bei Jürgen Osterhammel: Transferanalyse und Vergleich im Fernverhältnis. In: Hartmut Kaelble/Jürgen Schriewer (Hg.): Vergleich und Transfer. Komparatistik in den Sozial-, Geschichts- und Kulturwissenschaften. Frankfurt/New York 2003. S. 439-466, hier S. 450 f. 